\title{
Probabilistic Atlas Based Segmentation Using Affine Moment Descriptors and Graph-Cuts
}

\author{
Carlos Platero, Victor Rodrigo, Maria Carmen Tobar, Javier Sanguino, \\ Olga Velasco, and José M. Poncela \\ Applied Bioengineering Group - Technical University of Madrid \\ carlos.platero@upm.es
}

\begin{abstract}
We show a procedure for constructing a probabilistic atlas based on affine moment descriptors. It uses a normalization procedure over the labeled atlas. The proposed linear registration is defined by closed-form expressions involving only geometric moments. This procedure applies both to atlas construction as atlas-based segmentation. We model the likelihood term for each voxel and each label using parametric or nonparametric distributions and the prior term is determined by applying the vote-rule. The probabilistic atlas is built with the variability of our linear registration. We have two segmentation strategy: a) it applies the proposed affine registration to bring the target image into the coordinate frame of the atlas or b) the probabilistic atlas is nonrigidly aligning with the target image, where the probabilistic atlas is previously aligned to the target image with our affine registration. Finally, we adopt a graph cut - Bayesian framework for implementing the atlas-based segmentation.
\end{abstract}

Keywords: probabilistic atlas, affine transformation, graph-cut.

\section{Introduction}

Much research has been developed to integrate prior knowledge into the segmentation task. We focus on prior knowledge of shape and appearance of the object of interest. These approaches requiere a modeling or training step before the actual segmentation takes place. These ideas find their root in the active shape models first introduced by Cootes et al [1] based on matching a shape model to an unseen image using landmarks. Later, there has been increasing interest in using level set-based representation for shape priors $[2,3,4]$, which avoids landmarks. However, segmentation techniques that rely on the optimization of the complex functionals require the adjustment of multiple parameters. Consequently, these methods suffer from sensitivity to the tuning process.

In medical images there is sometimes a weak relation between voxel data and the label assignment. In such cases, spatial information must be taken into account in the segmentation process. One well-validated approach relies on combining the segmentations obtained from non-rigid aligning multiple manually labeled atlas with the target image [5]. This method makes no use of the intensity information. Considering such information could improves the quality of 
the atlas segmentation. The probabilistic atlas is commonly used in the analysis of medical images, since it integrates a priori knowledge of the shape and the appearance.

To combine prior knowledge and some type of regularization based on the framework of a Markov random field (MRF) is a well established technique for the medical image segmentation [6]. In these approaches spatial information in terms of a probabilistic atlas and the contextual information are used to formulate a maximum a posteriori probability (MAP-MRF). Since Grey et al [7] proposed graph cuts as a generic method for estimating the maximum a posteriori, it has been widely used for optimization in this area. For the case of two labels, Greig constructed a graph with two terminal vertices such that the minimum cut provides a global optimal labeling. For the multi-label problem, Ishikawa [8] solved the minimization problem for energy functions with pairwise terms that are convex in the linearly ordered labels. Therefore, we adopt a graph cut - Bayesian framework for implementing the atlas-based segmentation.

The paper is organized as follows: in Section 2, we show the problem of the linear registration and our approach based on the image normalization. Section 3 describes the method for constructing a probabilistic atlas. Section 4 presents our framework for segmentation using the atlas information and the graph cuts for optimizing the posterior probability. Finally, in Section 5 , we apply our procedure to liver segmentation from CT images.

\section{Linear Registration Using Affine Moment Descriptors}

We present a procedure for generating a probabilistic atlas based on affine moment descriptors. It captures the variability of learning samples and tries to generalize for the segmentation task. Our first step is to align the training samples, in order to avoid artifacts due to different pose. Traditionally, the pose parameters have been estimated minimizing a energy functional, via gradient descent $[3,9]$.

Our approach considers a training set consisting of $N$ binary images $\left\{S_{i}\right\}_{i=1, \ldots, N}: \Omega \subset \mathbb{R}^{n} \rightarrow\{0,1\}, n=2$ or 3 . For the multi-label problem, manual segmentation images have to be converted to binary images. All images in the database are aligned with a single binary image as reference, $\tilde{S}_{r e f}$. The new aligned images are defined as $\tilde{S}_{i}=S_{i} \circ T_{i}^{-1}$, where $T_{i}$ is an affine transformation, given by the composition of a rotation, a scaling transformation and a translation. Equivalently, $S_{i}=\tilde{S}_{i} \circ T_{i}$. We propose a criterion for alignment based on a shape normalization algorithm [11]. It is only necessary to compute the first and second order moments. The first-order moments locate the centroid of the shape and the second-order moments characterize the size and orientation of the image. Given a binary image $S_{i}$, we compute the second-order moments matrix, and the image is rotated using the eigenvectors and it is scaled along the eigenvectors according to the eigenvalues of the second-order moment matrix of $S_{i}$ and $\tilde{S}_{r e f}$, where $\tilde{S}_{\text {ref }}$ is a normalized shape. Then, it is translated by the centroid. We do not consider the problem of reflection (for this see [10]). If we only 
consider moments up to order two, $S_{i}$ is approximated to an ellipse/ ellipsoid centered at the image centroid. The rotate angles and the axes are determined by the eigenvalues and the eigenvectors of the second-order moment matrix [11]. Let $R_{i}$ be the rotation matrix.

Let $\left\{\lambda_{j}^{r e f}\right\}_{j=1, \ldots, n}$ be the eigenvalues of the reference image $\tilde{S}_{r e f}$. We consider one of the following scale matrices: a) $W_{i}=\sqrt{\frac{\lambda^{r e f}}{\lambda^{i}}} \cdot I$ where $\lambda^{c}=\left(\prod_{j=1}^{n} \lambda_{j}\right)^{1 / n}$, $c=\{$ ref,$i\}$ and $I$ is the identity matrix or b) $W_{i}$ is a diagonal matrix where $w_{j, j}=\sqrt{\frac{\lambda_{j}^{r e f}}{\lambda_{j}^{i}}}$. In the first case it is a homothety, while in the second case the size fits in each principal axis as the reference. The first option is used for shape priors without privileged directions otherwise the second case is chosen. Finally, the affine transformation translates the origin of the coordinate system to the reference centroid $\bar{x}_{r e f}$. We denote the $i$-shape centroid as $\bar{x}_{i}$. The affine transformation is then defined as follows:

$$
T_{i}^{-1}(x)=R_{i} \cdot W_{i} \cdot\left(x-\bar{x}_{i}\right)+\bar{x}_{r e f} .
$$

This affine transformation aligns from $S_{i}$ to $\tilde{S}_{\text {ref }}$. Of course, it is a bijection if $\operatorname{det}\left(R_{i} \cdot W_{i}\right) \neq 0$. If we use a scaling identical in all directions, $\tilde{S}_{r e f}$ will be only a numeric artifact for the pose algorithm. The alignment error does not depend on the reference, $\widetilde{S}_{r e f}$. But when each principal axis is adjusted to the reference, the alignment error depends on the choice of the reference. We can not guarantee the optimal pose for any shape. But neither the gradient descent method guaranteed to find the optimum because there is not evidence that the proposed functionals are convex. Our procedure is fast and optimum if the shapes are closed to ellipses or ellipsoids.

\section{Construction of the Probabilistic Atlas}

Our framework is based on the Bayesian decision theory. Given the target image to be segmented, $I: \Omega \subset \mathbb{R}^{n} \rightarrow \mathbb{R}$, and the probabilistic atlas, it assigns the label that maximizes the posterior probability:

$$
F_{x}=F(x)=\arg \max _{l_{j} \in L} p\left(I_{x} \mid l_{j}\right) p\left(x, l_{j}\right),
$$

where $F: \Omega \subset \mathbb{R}^{n} \rightarrow L=\left\{l_{1}, l_{2}, \ldots, l_{k}\right\}$ is a labeling of the voxels of $\Omega, p\left(I_{x} \mid l_{j}\right)$ represents the likelihood term of the voxel appearance at $x$ corresponding to the label $l_{j}$ and $p\left(x, l_{j}\right)$ is the prior term at $x$, which models the shape variability. Therefore, these terms represent the appearance and shape models and they are constructed using the aligned training images. The appearance and shape models are built with the variability of our linear registration.

\subsection{Appearance Prior Modeling}

The appearance model is obtained from the intensities of the voxels belonging to the set of aligned training images. Before building the appearance model, the 
training images in intensity are normalized using histogram matching. We denote the normalized and aligned training images as $\left\{\tilde{S}_{i}\right\}_{i=1, \ldots N}: \tilde{\Omega} \subset \mathbb{R}^{n} \rightarrow L$ and $\left\{\tilde{I}_{i}\right\}_{i=1, \ldots N}: \tilde{\Omega} \subset \mathbb{R}^{n} \rightarrow \mathbb{R}$. Gaussian mixture models are used intensively for distribution estimation and their parameters are tuned by using expectationmaximization based method [6], which provides a global view of the whole object appearance. In this work we model the probabilistic appearance for each voxel and each label using parametric or nonparametric distributions. We have implemented two options: i) each voxel on the aligned learning set follows a normal distribution for each label, $N\left(\mu\left(x, l_{j}\right), \sigma_{G}^{2}\left(x, l_{j}\right)\right)$ :

$$
\mu\left(x, l_{j}\right)=\frac{\sum_{\left\{i \mid \tilde{S}_{i}(x)=l_{j}\right\}} \tilde{I}_{i}(x)}{\#\left\{i \mid \tilde{S}_{i}(x)=l_{j}\right\}} \quad \sigma_{G}^{2}\left(x, l_{j}\right)=\frac{\sum_{\left\{i \mid \tilde{S}_{i}(x)=l_{j}\right\}}\left(\tilde{I}_{i}(x)-\mu\left(x, l_{j}\right)\right)^{2}}{\#\left\{i \mid \tilde{S}_{i}(x)=l_{j}\right\}} .
$$

ii) It follows a nonparametric distribution, considering the probabilistic atlas and the target image into the same coordinate frame (see next section):

$$
p\left(\tilde{I}_{x} \mid l_{j}\right)=\frac{1}{\#\left\{i \mid \tilde{S}_{i}(x)=l_{j}\right\} \sigma_{W}\left(x, l_{j}\right)} \sum_{\left\{i \mid \tilde{S}_{i}(x)=l_{j}\right\}} K\left(\frac{\tilde{I}(x)-\tilde{I}_{i}(x)}{\sigma_{W}\left(x, l_{j}\right)}\right),
$$

where $K(z)=\frac{1}{\sqrt{2 \pi}} \exp \left(-\frac{|z|^{2}}{2}\right)$ and

$\sigma_{W}^{2}\left(x, l_{j}\right)=\frac{1}{\#\left\{i \mid \tilde{S}_{i}(x)=l_{j}\right\}} \sum_{\left\{p \mid \tilde{S}_{p}(x)=l_{j}\right\}} \min _{p \neq q}\left(\tilde{I}_{p}(x)-\tilde{I}_{q}(x)\right)^{2}$.

\subsection{Shape Prior Modeling}

To capture the variability of the shape, the set of aligned manually segmented images are used. In $[2,3,4]$, principal component analysis (PCA) of the signed distance functions of training data is used to capture it. However, PCA provides a global view of the shape variability. Saad et al [12] introduce a modification of the idea of a probabilistic atlas by incorporating additional information derived from the distance transform. However, we have observed that a local estimation over our aligned training data provides robust results. It defines the prior term at $x$ applying the vote-rule as

$$
\sum_{j=1}^{k} p\left(x, l_{j}\right)=1 \quad p\left(x, l_{j}\right)=\frac{\#\left\{i \mid \tilde{S}_{i}(x)=l_{j}\right\}}{N}
$$

\section{Image Segmentation Strategy}

The appearance and shape models are built with the variability of our linear registration, without learning based on non-rigid registration as in [5]. The drawback is the need of an initial solution for the segmentation. However, this initial solution does not need to be robust because the proposed affine transformation uses only the first and second order moments.

Given a new image to be segmented, $I$, and an initial binary solution, $S$ : $\Omega \subset \mathbb{R}^{n} \rightarrow\{0,1\}$, we have two procedures: a) we apply the proposed affine 
registration to bring this image into the coordinate frame of the atlas $\tilde{S}=S \circ T^{-1}$ and $\tilde{I}=I \circ T^{-1}$, where $T^{-1}$ is calculated as (1) or b) the probabilistic atlas is nonrigidly aligning with the target image, where the probabilistic atlas is previously aligned to the target image with our affine registration, $S_{r e f}=\tilde{S}_{r e f} \circ T$. In both cases, the posterior probability is calculated for the unseen image.

Optimizing the posterior probability is not an easy task, especially because there are so many realizations of the MRF model and the optimization is prone to be caught in local maximums. Greig et al. [7] were the first to discover that powerful min-cut/max-flow algorithms from combinatorial optimization can be used to minimize certain important energy functions in computer vision. In particular, they showed that graph cuts can be used for restoration binary images. The problem was formulated as a maximum a posterior estimation with a MRF regularization that required the minimization of the following energy:

$$
E(F)=\sum_{x \in \Omega} V_{x}\left(F_{x}\right)+\sum_{\substack{\{x, y\} \\ x, y \in \Omega, x \neq y}} V_{x y}\left(F_{x}, F_{y}\right),
$$

where for the case of two labels $L=\left\{l_{1}, l_{2}\right\}, V_{x}\left(F_{x}\right)=\left\{\begin{array}{ll}\lambda_{x} & \text { if } F_{x}=l_{2} \\ 0 & \text { if } F_{x}=l_{1}\end{array}, \lambda_{x}=\right.$ $\log \left(\frac{p\left(I_{x} \mid l_{2}\right) p\left(x, l_{2}\right)}{p\left(I_{x} \mid l_{1}\right) p\left(x, l_{1}\right)}\right)$ and $V_{x y}\left(F_{x}, F_{y}\right)=\left\{\begin{array}{ll}\beta_{x y}>0 & \text { if } F_{x} \neq F_{y} \\ 0 & \text { if } F_{x}=F_{y}\end{array}\right.$. Greig constructed a graph with two terminal vertices $\{s, t\}$, such that the minimum cut provides a global optimal labeling. There is a directed edge $\{s, x\}$ from $s$ to the voxel $x$ with weight $\omega_{s x}=\lambda_{x}$ if $\lambda_{x}>0$; otherwise, there is a directed edge $\{x, t\}$ from $x$ to $t$ with weight $\omega_{x t}=-\lambda_{x}$. There is an undirected edge $\{x, y\}$ between two internal vertices with weight $\omega_{x y}=\beta_{x y}$. It is a smoothness term based on intensities $\{I(x), I(y)\}$, which represents the realizations of the MRF model. For the multi-label problem, if each $V_{x y}$ defines a metric, then minimizing (2) is known as the metric labeling problem and can be optimized effectively with the $\alpha$-expansion algorithm [13].

\section{$5 \quad$ Validation, Experiments and Results}

The experimental validation is performed using the problem of liver segmentation from 3D CT images. Algorithms relying solely on image intensities or derived features usually fail. To deal with missing or ambiguous low-level information, shape and appearance prior information has to be employed. The proposed method has been considered on 20 patients CT slice set and tested on another 10 specified CT datasets.

In a first step we align the training data by the proposed procedure. In this case, each principal axis is adjusted to the reference. Experimentally, $\tilde{S}_{\text {ref }}$ was chosen by minimizing the Similarity Index, $\left(S I=\frac{1}{N} \sum_{i} \frac{\left(S_{i} \circ T_{i}^{-1}\right) \cap \tilde{S}_{r e f}}{\left(S_{i} \circ T_{i}^{-1}\right) \cup \tilde{S}_{r e f}}\right)$, over the training set. We compare our approach with other techniques. Table 1 lists the mean $\left(\mu_{S I}\right)$ and standard deviation $\left(\sigma_{S I}\right)$ values of the $S I$ metric over the training data set. Given a CT abdominal image as target image, our approach starts 
Table 1. Results of the affine registration: $\mu_{S I}$ and $\sigma_{S I}$ values of the $S I$

\begin{tabular}{|c|c|c|c|}
\hline \multicolumn{4}{|c|}{ Type SSD [3] MI [9] Our } \\
\hline$\mu_{S I}$ & 0.54 & 0.63 & 0.68 \\
\hline$\sigma_{S I}$ & 0.10 & 0.07 & 0.05 \\
\hline
\end{tabular}

with an initial solution. It is obtained filtering the image by a nonlinear diffusion filter with selection of the optimal stopping time. Then, region growing and 3D edge detector are applied to the filtered image. Morphological post-processing merges the previous steps, giving the initial solution. Next, the probabilistic atlas and the target image are placed into the same coordinate frame using our affine transformation. The non-rigid registration between the probabilistic atlas and the target image was performed with ElastiX [14]. We use the min-cut/max flow algorithm of Boykov-Kolmogorov for energy minimization [15]. In our implementation, the data term, defines the edge weights connecting each node to the source $s$ and $\sin k t$, is proposed to: $\lambda_{x}=\log \left(\frac{\frac{p\left(I_{x} \mid l_{2}\right) p\left(x, l_{2}\right)}{\sigma\left(x, l_{2}\right)}}{\frac{p\left(I_{x} \mid l_{1}\right)\left(x, l_{1}\right)}{\sigma\left(x, l_{1}\right)}}\right)$. This proposal is based on the more reliable of the probability estimations if there are less dispersion in the samples. We have experimentally observed that normal distribution model for probabilistic appearance prior is more robust than the non-parametric one. We think that it is due to less dependence on the initial solution. The parameters of $V_{x y}$ were tuned using the leave-one-out technique from training data according to the segmentation scores. In our case, a 6 neighborhood relation is used to save memory.

Fig. 1 shows slices from two cases, drawing the result of the method (in blue) and the reference (in red). The quality of the segmentation and its scores are based on the five metrics [16]. Each metric was converted to a score where 0 is the minimum and 100 is the maximum. Using this scoring system one can loosely say that 75 points for a liver segmentation is comparable to human performance.

Table 2. Average values of the metrics and scores for all ten test case: volumetric overlap error $\left(m_{1}\right)$, relative absolute volume difference $\left(m_{2}\right)$, average symmetric surface distance $\left(m_{3}\right)$, root mean square symmetric surface distance $\left(m_{4}\right)$ and maximum symmetric surface distance $\left(m_{5}\right)$

\begin{tabular}{|l||c||c|c|c|c|c|}
\hline Type & $m_{1}$ & $m_{2}$ & $m_{3}$ & $m_{4}$ & $m_{5}$ \\
\hline \multirow{2}{*}{ AT1 [5] } & metrics & $12.5 \%$ & $3.5 \%$ & $2.41 \mathrm{~mm}$ & $4.40 \mathrm{~mm}$ & $32.4 \mathrm{~mm}$ \\
\cline { 2 - 7 } & scores & 51 & 80 & 40 & 40 & 57 \\
\hline \hline \multirow{2}{*}{ Affine } & metrics & $12.1 \%$ & $2.5 \%$ & $1.71 \mathrm{~mm}$ & $2.96 \mathrm{~mm}$ & $26.1 \mathrm{~mm}$ \\
\cline { 2 - 7 } & scores & 53 & 87 & 57 & 59 & 66 \\
\hline \hline \multirow{2}{*}{ Nonrigid } & metrics & $9.69 \%$ & $3.9 \%$ & $1.12 \mathrm{~mm}$ & $2.03 \mathrm{~mm}$ & $22.1 \mathrm{~mm}$ \\
\cline { 2 - 7 } & scores & 62 & 79 & 72 & 72 & 71 \\
\hline
\end{tabular}




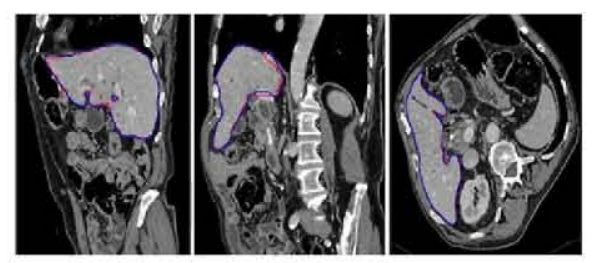

(a)

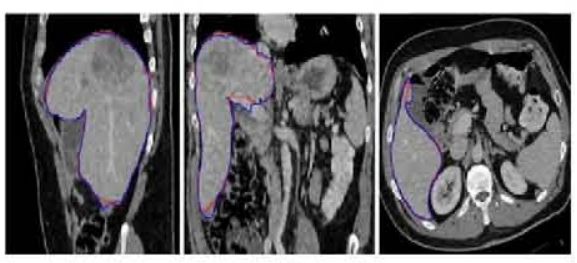

(b)

Fig. 1. From left to right, a sagittal, coronal and transversal slice for an easy case (a) and a difficult one (b). The outline of the reference standard segmentation is in red, the outline of the segmentation of the method described in this paper is in blue.

Table 2 lists the average values of the metrics and their scores over the test data set. It shows the performances for the three segmentation strategies: a) atlas matching b) probabilistic atlas with only linear registration and b) probabilistic atlas with nonrigid techniques. The average computation times for the liver segmentation task are 203.4 s., 25.3 s. and 211.7 s. respectively ([Dual CPU] Intel Xeon E5520 @ 2.27GHz).

\section{Conclusion}

We have presented two main contributions. Firstly, the linear registration has been solved using an image normalization procedure applied to the labeled atlas. An advantage is that the proposed affine transformation is defined by closed-form expressions involving only geometric moments. No additional optimization over pose parameters is necessary. This procedure has been applied both to atlas construction as atlas-based segmentation. Secondly, we model the probabilistic appearance for each voxel and each label using parametric or nonparametric distributions and the prior term is determined by applying the vote-rule. The appearance and shape models are built with the variability of proposed linear registration. We adopt a graph cut - Bayesian framework for implementing the atlas-based segmentation. Finally, we illustrate the benefits of our approach on the liver segmentation from CT images.

\section{References}

1. Cootes, T., Taylor, C., Cooper, D., Graham, J., et al.: Active shape models-their training and application. Computer Vision and Image Understanding 61, 38-59 (1995)

2. Leventon, M., Grimson, W., Faugeras, O.: Statistical shape influence in geodesic active contours. In: IEEE Computer Society Conference on Computer Vision and Pattern Recognition, vol. 1. IEEE Computer Society, Los Alamitos (1999/2000)

3. Tsai, A., Yezzi, A., Wells, W., Tempany, C., Tucker, D., Fan, A., Grimson, W., Willsky, A.: A shape-based approach to the segmentation of medical imagery using level sets. IEEE Transactions on Medical Imaging 22, 137-154 (2003) 
4. Cremers, D., Rousson, M.: Efficient kernel density estimation of shape and intensity priors for level set segmentation. In: Suri, J.S., Farag, A. (eds.) Parametric and Geometric Deformable Models: An application in Biomaterials and Medical Imagery. Springer, Heidelberg (2007)

5. Wang, Q., Seghers, D., D'Agostino, E., Maes, F., Vandermeulen, D., Suetens, P., Hammers, A.: Construction and validation of mean shape atlas templates for atlas-based brain image segmentation. In: Christensen, G.E., Sonka, M. (eds.) IPMI 2005. LNCS, vol. 3565, pp. 689-700. Springer, Heidelberg (2005)

6. Park, H., Bland, P., Meyer, C.: Construction of an abdominal probabilistic atlas and its application in segmentation. IEEE Transactions on Medical Imaging 22, 483-492 (2003)

7. Greig, D., Porteous, B., Seheult, A.: Exact maximum a posteriori estimation for binary images. Journal of the Royal Statistical Society. Series B (Methodological) 51, 271-279 (1989)

8. Ishikawa, H.: Exact optimization for Markov random fields with convex priors. IEEE Transactions on Pattern Analysis and Machine Intelligence 25, 1333-1336 (2003)

9. Maes, F., Collignon, A., Vandermeulen, D., Marchal, G., Suetens, P.: Multimodality image registration by maximization of mutual information. IEEE Transactions on Medical Imaging 16, 187-198 (2002)

10. Heikkila, J.: Pattern matching with affine moment descriptors. Pattern Recognition $37,1825-1834$ (2004)

11. Pei, S., Lin, C.: Image normalization for pattern recognition. Image and Vision Computing 13, 711-723 (1995)

12. Saad, A., Hamarneh, G., Moller, T.: Exploration and Visualization of Segmentation Uncertainty Using Shape and Appearance Prior Information. IEEE Transactions on Visualization and Computer Graphics 16 (2010)

13. Boykov, Y., Veksler, O., Zabih, R.: Fast approximate energy minimization via graph cuts. IEEE Transactions on Pattern Analysis and Machine Intelligence, 1222-1239 (2001)

14. Klein, S., Staring, M., Murphy, K., Viergever, M., Pluim, J.: elastix: a toolbox for intensity-based medical image registration. IEEE Transactions on Medical Imaging $29(2010)$

15. Boykov, Y., Kolmogorov, V.: An experimental comparison of min-cut/max-flow algorithms for energy minimization in vision. IEEE Transactions on Pattern Analysis and Machine Intelligence 26, 1124-1137 (2004)

16. van Ginneken, B., Heimann, T., Styner, M.: 3D segmentation in the clinic: A grand challenge. In: Proceedings of MICCAI Workshop on 3D Segmentation in the Clinic: A Grand Challenge, pp. 7-15 (2007) 